\title{
An Alternative Maze to Assess Novel Object Recognition in Mice
}

José Fernando Oliveira da Cruz ${ }^{1,2, \$ a}$, Maria Gomis-Gonzalez ${ }^{3}$, Rafael Maldonado ${ }^{3}$, Giovanni Marsicano ${ }^{1,2}$, Andrés Ozaita ${ }^{3}$ and Arnau Busquets-Garcia ${ }^{1,2, \$ b, ~ * ~}$

${ }^{1}$ INSERM, U1215 NeuroCentre Magendie, 33000 Bordeaux, France; ${ }^{2}$ University of Bordeaux, 33000 Bordeaux, France; ${ }^{3}$ Laboratory of Neuropharmacology, Department of Experimental and Health Sciences, Universitat Pompeu Fabra, Barcelona, Spain; IMIM Hospital Del Mar Research Institute,

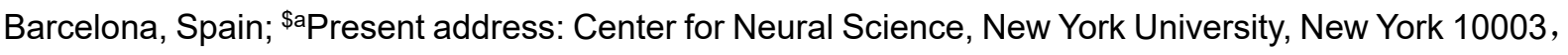
USA; \$bPresent address: Integrative Pharmacology and Systems Neuroscience Research Group, Neurosciences Research Program, IMIM (Hospital del Mar Medical Research Institute), Dr. Aiguader 88, Barcelona, 08003, Spain

*For correspondence: abusquets@imim.es

[Abstract] The novel object recognition (NOR) task is a behavioral test commonly used to evaluate episodic-like declarative memory and it relies on the innate tendency of rodents to explore novelty. Here we present a maze used to evaluate NOR memory in mice that reduces the time of the assay while improving reliability of the measurements by increasing the exploratory behavior. This memory test, being performed in a two-arms maze, is suitable for several strains of mice (including inbreed and outbreed) and does not require extended training sessions allowing an accurate temporal assessment of memory formation. This particular maze increases the mouse exploration time and reduces variability compared to other arenas used before to assess NOR. As both long- and short-term NOR memory can be easily and accurately quantified using this paradigm, this improved methodology can be easily applied to study pharmacological, genetic or age-related modulation of cognitive function.

Keywords: Novel Object recognition memory, Cognition, Mouse behavior, Memory task, Learning, Animal behavior

[Background] Novel object recognition (NOR) memory task is a commonly used experimental behavioral task aimed at studying learning and memory (Ennaceur, 2010). The main postulate behind this behavioral task is that, in the presence of a novel and a previously presented (familiar) object, rodents increase their exploration towards the novel object (Ennaceur and Delacour, 1988). The increased exploration of the novel object is interpreted as indirect evidence that animals acquired a memory of the familiar object, and thus increase their exploration to the novel one. As a consequence, NOR paradigm is considered a reliable model to test hippocampal and temporal lobe function, as lesions within these brain regions abolish recognition memory (Winters et al., 2008; Broadbent et al., 2010).

There are several advantages of using the NOR task. First, NOR task takes advantage of the animal's tendency to approach and explore novelty. Therefore, this task does not require preliminary extended training and training can happen in a single trial session, allowing a robust temporal definition of the different stages of the memory formation (i.e., acquisition, consolidation, re-consolidation and retrieval). 
Also, it does not require exposure to aversive or stressful stimuli stronger than novelty itself, nor it requires food or water restriction. Altogether, these factors have contributed to the growing popularity of this behavioral paradigm (Dere et al., 2007).

The NOR task has been replicated using a variety of environmental designs and objects in rodents. This task has been classically performed in a large open field where rodents navigate to explore the objects (Bevins and Besheer, 2006) or in a Y-shaped maze with very short arms were rats are exposed to two objects that they explore without the need of extensive exploration (Winters et al., 2004). NOR tasks that involve an open field environment require longer habituation periods (several days) that must be performed during several consecutive training sessions (Dere et al., 2007). As a consequence, the evaluation of long-term NOR memory seems less reliable (Sik et al., 2003). One important limitation of the open field setting is increased variability within similar experimental groups observed in terms of the interaction/exploratory time of the animal with the object. The high variability can be caused by spatial and contextual confounds together with the pro-anxiogenic effects of the environment (open field) (Hale et al., 2008). Overall, this lengthens the overall assay, reduces the accuracy of the test and entails the use of a higher number of animal in each experimental group. On the other hand, the Y-shaped maze used with rats, hinders the assessment of the locomotor activity of the animals, which in turn could bias the outcome of the test due to the short length of the corridors where the object exploration takes place.

Here, we propose a maze consisting in two arms separated by 90 degrees that heen equally called in previous publications as L- or V-Maze (Puighermanal et al., 2009 and 2013; Busquets-Garcia, et al., 2011, 2013, 2016 and 2018; Aso et al., 2012; Hebert-Chatelain et al., 2016; Aloisi et al., 2017). This maze has long corridors to minimize the context surrounding the objects and to reduce the weight of other possible cues rather than the objects themselves. These features direct the animal's exploratory behavior towards the objects and facilitates the interaction of the animal with the object. Moreover, the arms in this maze are relatively narrow, thus reducing the possible anxiety-related bias of the open field environment. Altogether, this version of the maze maximizes the exploration time of the objects relative to the surrounding context and increases the accuracy of the test. This improved setting has been now successfully used to evaluate short- and long-term memory depending on the retention time defined between the training session and the test session ( $3 \mathrm{~h}$ and $24 \mathrm{~h}$, respectively) in both inbreed (C57BL/6) and outbreed (CD-1) mice (Puighermanal et al., 2009 and 2013; Aso et al., 2012; Busquets-Garcia et al., 2011, 2013, 2016-and 2018; Hebert-Chatelain et al., 2016; Aloisi et al., 2017). Also, it improves both the variability of the assay and the reproducibility, which in turn reduces the number of mice required, the length of the sessions and the overall complexity of the protocol. However, this new NOR protocol have the limitation that it has to be adapted (e.g., light intensity, room noise, room space or objects used) every time that it is used in a novel mouse strain or when it is set up in a new animal space. Thus, it needs a pilot experiment to verify all these parameters and it might not be as flexible as an open field arena. Sor far, this specific NOR task has been used in access memory performance in a mouse model of Alzheimer (8 months or 18 months) (Aso et al., 2012), to study the acute effects of stress on recognition memory (Busquets-Garcia et al., 2016), the memory deficits in a mouse model of Fragile $X$ syndrome (Busquets-Garcia et al., 2013; Aloisi et al., 2017) and the modulation of memory by the 
endocannabinoid system (Puighermanal et al., 2009 and 2013; Hebert-Chatelain et al., 2016; BusquetsGarcia et al., 2018; Robin et al., 2018; Oliveira da Cruz et al., 2019).

\section{Materials and Reagents}

1. Disposable paper towels

2. Mice: 8-12 weeks old C57BL/6J and/or CD1 mice (Charles River, France or any company that provide mice for laboratories)

3. Water

4. $70 \%$ ethanol

\section{Equipment}

1. The NOR maze (see Figure 1)

We have designed and built this maze in the lab. The material used to build the maze was black or dark grey matt plexiglas.
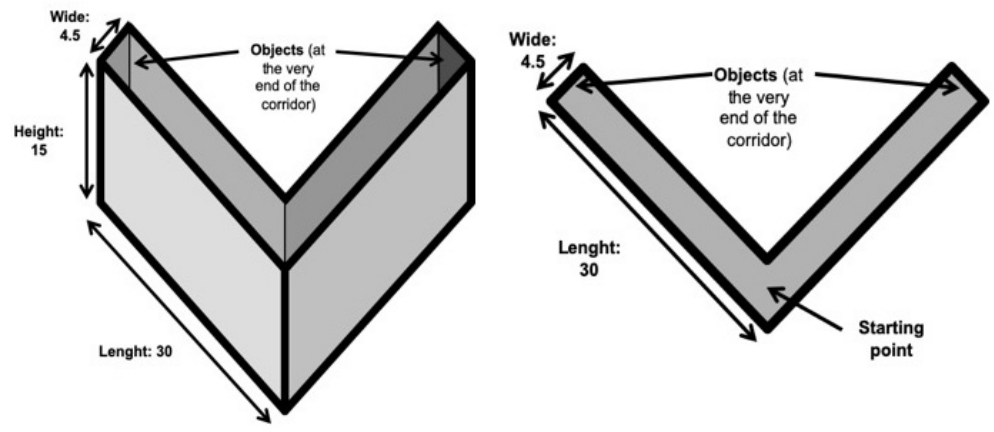

Figure 1. Maze to assess NOR memory. Dimensions are in $\mathrm{cm}$.

2. Two pairs of objects (see below for information regarding the objects)

These pairs of objects must be first validated in mice (see Procedure) to guarantee no intrinsic preference or aversion.

3. Experimental room with adjustable light (30-50 lux)

4. Real-time video acquisition system

5. Casual Stopwatches or any computer application to count the exploration time

\section{Software}

1. Any video Acquisition Software

2. Behavior Scoring Panel-(c) 2008 by A. DUBREUCQ Version 3.0 beta. A home-made software that allow us counting the exploration time. 
Note: If possible and to avoid any confounding factor, it is recommended that the experimenter is not present in the same room where the mouse is performing the task.

\section{Procedure}

A. Habituation session

This step is performed to familiarize mice with the maze. The habituation session lasts for 9 min.

1. Turn on the video recording system and set the parameters for data acquisition: Make sure that there is constant uniform lightning conditions in the maze (areas of interest, contrast for mouse detection, etc.) and in the room throughout the session (habituation, training and test sessions).

2. Place the animal in the maze without objects at the starting point (crossing-point between both arms, Figure 1).

3. At the end of the session, remove the animal from the maze and return it to its home-cage.

4. Then, clean maze and the objects with $70 \%$ ethanol and move to the next animal (back to Step A2).

Note: In order to avoid that odor cues from previous mice alter mouse exploration, it is crucial to carefully clean the surface of the maze and objects between each session with a $70 \%$ ethanol solution.

\section{B. Training session}

The training session is performed $24 \mathrm{~h}$ after the habituation session. The training session lasts for $9 \min$.

1. Turn on the video recording and data acquisition system.

2. Place two identical objects in the extremities of the maze in direct contact with the wall (see Figures 1 and 2).

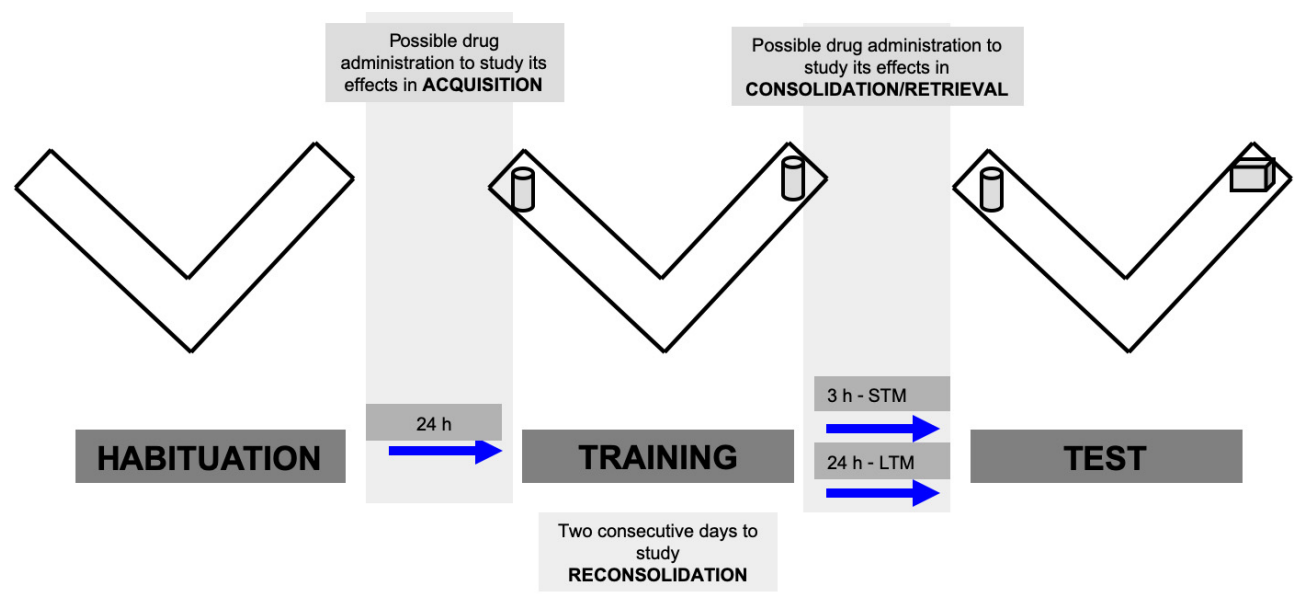

Figure 2. Scheme of the object recognition protocol. There are three different sessions (habituation, training and test) and each session lasts $9 \mathrm{~min}$. Short- or long-term memory can be assessed depending on the inter-session interval between the training and the test session. 
3. Place the animal at the starting point facing towards the edge of the two arms (crossing-point between the two arms).

4. Use the stopwatches, to record the time that the mouse spends exploring each object (see below). During this session, rodents usually spend around $50 \%$ of the total exploratory time for each one of the objects.

5. At the end of the training session, remove the mouse from the maze and leave it undisturbed in its home-cage.

6. After cleaning the maze as explained in the "habituation session" of the maze, start the training session for the following animal.

\section{Pharmacological treatments}

Pharmacological treatments can be used to study cellular and molecular mechanisms involved in different memory phases of NOR. For instance, in order to test the effect of a particular pharmacological treatment on memory acquisition (Figure 2), the drug must be administered before the training session. The administration time must be adjusted by taking in consideration the pharmacokinetics of the substance. Moreover, it is important to verify that the drug has no locomotor effects as it may influence the task outcome independently of the memory performance (e.g., impaired locomotor activity may affect exploration and consequently the capacity of the animal to move towards the objects to explore them). To study the effect of a pharmacological treatment on memory consolidation, the drug should be administered after the training session. Finally, to assess memory re-consolidation, mice are re-exposed to the same training conditions (same combination of objects during a 9 min session) $24 \mathrm{~h}$ after the first training session and the drug can be administered before or after the second training session.

\section{Testing phase}

The test session could be performed $3 \mathrm{~h}$ after training to evaluate short-term memory, or $24 \mathrm{~h}$ after training to evaluate long-term memory. The training session lasts for 9 min.

1. Turn on the video recording and data acquisition system.

2. Clean the maze.

3. Place one of the familiar objects at the end of one arm and the novel object at the end of the other arm. The position of the novel object (i.e., right vs. left arm) must be counterbalanced in between mice to avoid any possible confounding cue in the room that results in a preference for one of the arms of the maze.

4. Place the animal at the starting point, as previously mentioned above.

5. As in the previous sessions (habituation and training sessions), the observation is accomplished by a closed-circuit camera attached to a recording system to avoid unnecessary stress or uncontrolled cues to the animal (see Video 1). 


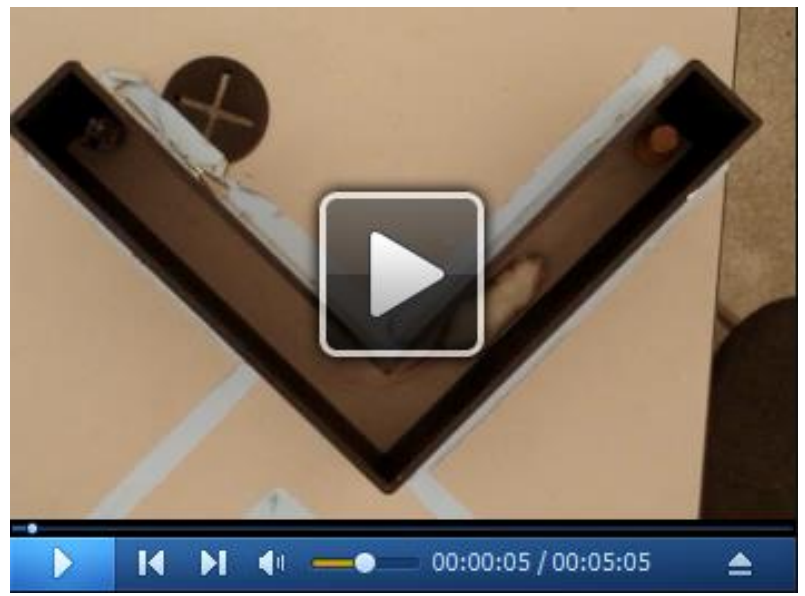

Video 1. Representative video showing object exploration in both arms of the maze

6. Use two stopwatches and assign each stopwatch to record the exploration time for each object. The experimenter, blind to the experimental conditions, measures the exploration time for each object analyzing the image obtained by the closed-circuit camera. Simultaneously, the video is recorded for documentation purposes together with video-tracking of general activity (time spent in each arm, number of entries in each arm and distance travelled).

7. At the end of the test session, remove the animal from the maze and return it to the home-cage. After cleaning the maze with water, start with another animal (back to Step C3).

8. After the session, export the experimental data and analyze the results (see Data analysis).

Note: If the total exploration time is very low $(<5-10 \mathrm{~s})$ in the training and/or test sessions the results of these mice are not considered for the experiment. This may happen due to:

a. The object is not adequate for the task (i.e., it may induce stress or anxiety to the animals). Verify that the object was properly cleaned as it could contain the odors of a previous animal and re-evaluate the objects to understand if there is intrinsic preference or aversion.

$b$. The animal does not explore the maze because of a locomotor deficit or it does not explore the maze due to a pharmacological treatment and/or a genetic manipulation. If there is a clear locomotor alteration during the performance of the task, exclude the animal from the analysis.

\section{Data analysis}

A. Quantification of object exploration

Exploratory time is scored manually by an experimenter blind to the experimental design during the training and test sessions. The manual scoring is preferred due to the current difficulty for automated systems to accurately discriminate between tail and head and to correctly count exploration time. Nevertheless, we acknowledge that deep-learning based approaches for pose estimation (Mathis et al., 2018; Nath et al., 2019) may provide a reliable way to automatize exploration quantification in the near future. 
Exploration of an object is scored when: the animal nose is facing directly the object $(<2 \mathrm{~cm})$ as described before (Robin et al., 2018; Oliveira da Cruz et al., 2019). Exploration is not scored when: the animal does not interact with the object or it stands on the top of the object without facing the object.

Note: If the animal does not interact with both objects at least once during the complete duration of the session, the session is not valid.

B. Analysis of Object Exploration

In order to evaluate differences in the exploration during this task when comparing two or more experimental groups, 2 types general analysis can be performed: 1) Discrimination Index (DI); 2) Familiar vs. Novel analysis. In addition, the total exploration time is also expressed to control variability in overall exploration between experimental groups. The DI is calculated with the following formula:

$\mathrm{DI}=\left[\right.$ Timenovel $_{-}-$Timefamiliar $\left.)\right] /\left(\left(\right.\right.$Time $\left._{\text {familiar }}\right)+\left(\right.$ Time $\left.\left._{\text {novel }}\right)\right]$

Additional variables can be measured: time spent in each arm, number of entries in each arm and distance travelled. Exploration data can be represented in mean \pm SEM. Two-way Student's $t$-test and one-way ANOVA statistical analysis are the statistical tests used in these experiments. Comparisons are considered statistically significant when the level of significance is lower than 0.05 .

\section{Expected Results}

In the experimental conditions reported in this protocol, the general exploration time observed is around 15-40 s for each object during a typical training session. If the mice discriminate between the familiar and the novel object, the exploration time in the test session will be around 10-35 s for the familiar object and around 30-60 s for the novel object (Figure 3 and Table 1). If the recognition memory is impaired, as we described in Tetrahydrocannabinol (THC)-injected mice (Puighermanal et al., 2009), mice will explore the same amount of time both novel and familiar objects during the test session (20-40 s), and so they will not discriminate between them (Figure 3 and Table 1).
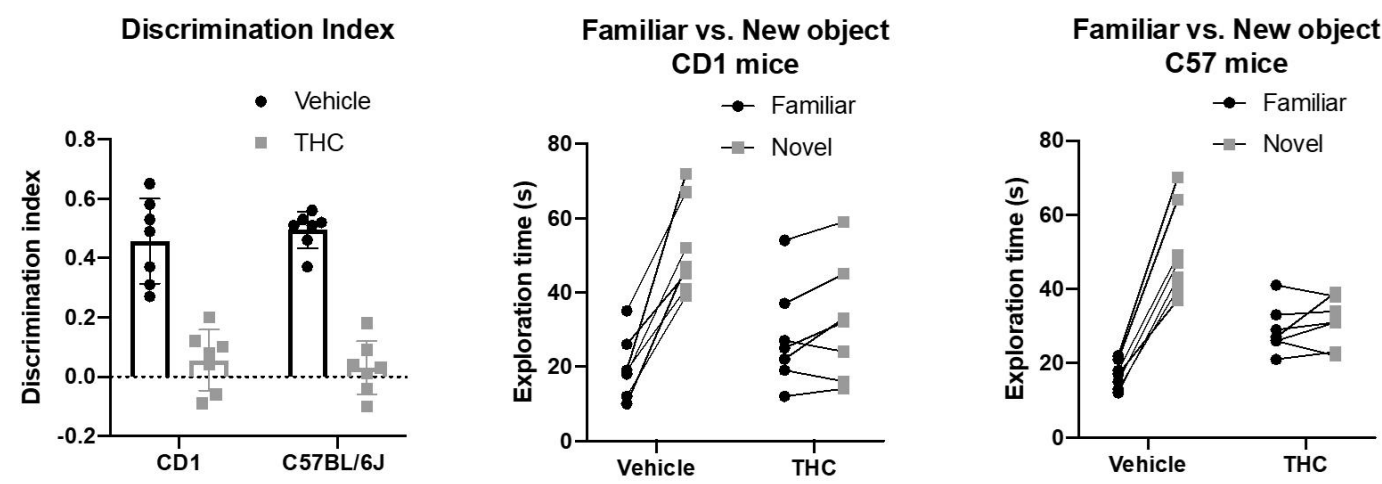

Figure 3. Representative graph of an example of the NOR task. Further details in Table 1. 
Please cite this article as: Oliveira da Cruz et. al., (2020). An Alternative Maze to Assess Novel Object Recognition in Mice,Bio-protocol 10 (12): e3651. DOI:

10.21769/BioProtoc. 3651.

\section{bio-protocol}

Table 1. An example of the expected data and analysis. Representative example of an experiment using a pro-amnesic dose of delta9tetrahydrocannabinol (THC, $10 \mathrm{mg} / \mathrm{kg}$, i.p., light colour) in CD1 and C57BL/6J mice, or its vehicle (VEH, dark colour) as control, administered after the training session. The exploration time (seconds) in the familiar object $(\mathrm{F})$; the exploration time on the second object in the training session $\left(\mathrm{F}^{\prime}\right)$; the exploration time in the novel object $(\mathrm{N})$; the total exploration time $(\mathrm{ET}=\mathrm{F}+\mathrm{F}$ ' or $\mathrm{ET}=\mathrm{F}+\mathrm{N})$; the percentage of exploration time devoted to each object during the training and the test sessions $\left(\% \mathrm{ET}=[\mathrm{F} / \mathrm{ET}] \times 100 ; \% \mathrm{ET}=\left[\mathrm{F}^{\prime} / \mathrm{ET}\right] \times 100 ; \% \mathrm{ET}=[\mathrm{N} / \mathrm{ET}] \times 100\right)$; the discrimination index is calculated for the test session $(\mathrm{DI}=[\mathrm{N}-\mathrm{F}] /[\mathrm{N}+\mathrm{F}])$.

\begin{tabular}{|c|c|c|c|c|c|c|c|c|c|c|c|c|c|c|c|}
\hline \multicolumn{8}{|c|}{ CD1 mice } & \multicolumn{8}{|c|}{ C57BL/6J } \\
\hline \multirow[b]{2}{*}{ Mouse } & \multicolumn{3}{|c|}{ Training } & \multicolumn{4}{|c|}{ Test } & \multirow[b]{2}{*}{ Mouse } & \multicolumn{3}{|c|}{ Training } & \multicolumn{4}{|c|}{ Test } \\
\hline & $\mathbf{F}$ & $F^{\prime}$ & ET & $\mathbf{F}$ & $\mathbf{N}$ & ET & DI & & $\mathbf{F}$ & $F^{\prime}$ & ET & $\mathbf{F}$ & $\mathbf{N}$ & ET & DI \\
\hline 1 & 42 & 45 & 87 & 35 & 67 & 102 & 0.31 & 1 & 55 & 45 & 100 & 18 & 49 & 67 & 0.46 \\
\hline 2 & 54 & 36 & 90 & 19 & 41 & 60 & 0.37 & 2 & 27 & 23 & 50 & 22 & 70 & 92 & 0.52 \\
\hline 3 & 39 & 34 & 73 & 12 & 39 & 51 & 0.53 & 3 & 16 & 18 & 34 & 12 & 43 & 55 & 0.56 \\
\hline 4 & 40 & 36 & 76 & 18 & 52 & 70 & 0.49 & 4 & 21 & 24 & 45 & 13 & 40 & 53 & 0.51 \\
\hline 5 & 53 & 58 & 111 & 26 & 45 & 71 & 0.27 & 5 & 25 & 24 & 49 & 15 & 47 & 62 & 0.52 \\
\hline 6 & 43 & 49 & 92 & 19 & 72 & 91 & 0.58 & 6 & 29 & 33 & 62 & 21 & 64 & 85 & 0.51 \\
\hline 7 & 61 & 56 & 117 & 10 & 47 & 57 & 0.65 & 7 & 44 & 42 & 86 & 17 & 37 & 54 & 0.37 \\
\hline Mean & 47.4 & 44.9 & 92.3 & 19.9 & 51.9 & 71.8 & 0.46 & Mean & 31 & 29.8 & 60.8 & 16.9 & 50 & 66.9 & 0.49 \\
\hline$\%$ ET & 51.4 & 48.6 & - & 27.7 & 72.3 & - & - & \%ET & 51 & 49 & - & 25.2 & 74.8 & - & - \\
\hline Mouse & $\mathbf{F}$ & $F^{\prime}$ & ET & $\mathbf{F}$ & $\mathbf{N}$ & ET & DI & Mouse & $\mathbf{F}$ & F' & ET & $\mathbf{F}$ & $\mathbf{N}$ & ET & DI \\
\hline 1 & 37 & 32 & 69 & 25 & 32 & 57 & 0.12 & 1 & 28 & 32 & 60 & 27 & 39 & 66 & 0.18 \\
\hline 2 & 39 & 46 & 85 & 22 & 33 & 55 & 0.2 & 2 & 34 & 28 & 62 & 26 & 22 & 48 & -0.10 \\
\hline 3 & 30 & 38 & 68 & 27 & 24 & 51 & -0.06 & 3 & 29 & 45 & 74 & 33 & 34 & 67 & 0.01 \\
\hline 4 & 51 & 52 & 103 & 54 & 59 & 113 & 0.04 & 4 & 35 & 28 & 63 & 21 & 23 & 44 & 0.04 \\
\hline 5 & 49 & 50 & 99 & 37 & 45 & 82 & 0.1 & 5 & 39 & 46 & 85 & 26 & 31 & 57 & 0.09 \\
\hline 6 & 57 & 42 & 99 & 19 & 16 & 35 & -0.09 & 6 & 45 & 51 & 96 & 29 & 31 & 60 & 0.03 \\
\hline
\end{tabular}


Please cite this article as: Oliveira da Cruz et. al., (2020). An Alternative Maze to Assess Novel Object Recognition in Mice,Bio-protocol 10 (12): e3651. DOI:

10.21769/BioProtoc.3651.

\section{biio-protocol}

www.bio-protocol.org/e3651

Bio-protocol 10(12): e3651

DOI:10.21769/BioProtoc.3651

\begin{tabular}{|c|c|c|c|c|c|c|c|c|c|c|c|c|c|c|c|}
\hline 7 & 43 & 51 & 94 & 12 & 14 & 26 & 0.08 & 7 & 29 & 38 & 67 & 41 & 38 & 79 & -0.04 \\
\hline Mean & 43.7 & 44.4 & 88.1 & 28 & 31.9 & 59.9 & 0.06 & Mean & 34.1 & 38.3 & 72.4 & 29 & 31.1 & 60.1 & 0.03 \\
\hline \%ET & 49.6 & 50.4 & - & 46.8 & 53.2 & - & - & \%ET & 47.1 & 52.9 & - & 48.3 & 51.7 & - & - \\
\hline
\end{tabular}




\section{$\underline{\text { Notes }}$}

1. Mice are housed for a minimum of 5 days in the experimental room where the behavioral task is set to take place. Animals are commonly housed collectively (3-6 animals per cage) in a saw dust environment with nesting material (cotton cube), water/food ad libitum and controlled temperature $\left(21 \pm 1^{\circ} \mathrm{C}\right)$ and humidity $(55 \pm 10 \%)$. Lighting is maintained at 12-h dark/light cycles (on at 7:00 a.m. and off at 7:00 p.m.) and experiments take place in the beginning of the light phase (typically 9:00 a.m.-2:00 p.m.). Home-cages should not be changed, and animals should not be disturbed during the previous days preceding the task as it may affect their performance. Housing facility should be located in the vicinity of the testing rooms as the stress and novelty of transport may alter exploration.

2. Choosing suitable objects is an essential step in this task. At least two distinct pairs of objects are needed. Features such as texture (stone, wood, plastic, metal) (Figure 4), color tonality and shape should be carefully considered when selecting objects to be used in this task. It is important to verify that animals spend a similar amount of time interacting with each object in the training session. Any preference or aversion for the objects should be avoided since it could represent an important bias for the interpretation of the results. In order to avoid this possible bias, preliminary experiments must be performed presenting to the animals both objects simultaneously and analyzing the exploration time for each one. The time exploring each object should be similar (around 50\% of the total exploration time devoted to each object) when both objects are presented for the first time to the mice. The exploration time for each object can be influenced by the shape and physical characteristics of the object. However, objects must be different enough from each other (color tonality, texture, shape) to facilitate the discrimination between both objects in the test session.

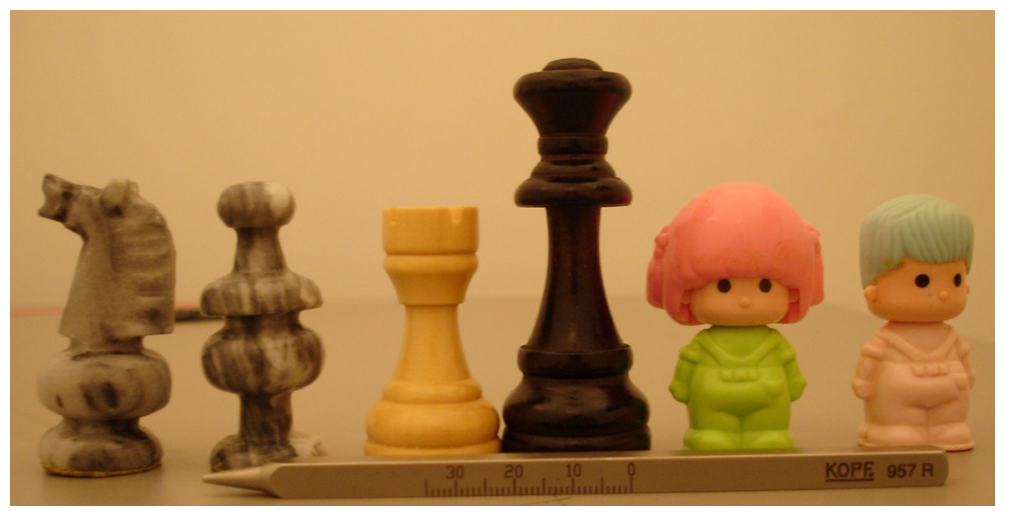

Figure 4. Objects used in our NOR paradigm. In this image you can find different examples of the objects used in our NOR paradigm. As you can see, different colors, shapes and materials can be used.

3. It is important to avoid odor cues and it is therefore mandatory to clean the maze and objects between animals and especially between experiments using different experimental conditions. 
4. It can happen that control animals do not show appropriate discrimination of the novel object. This can be due to a specific preference for one object or it could also be caused by the different, uncontrolled, conditions between the training and test sessions (e.g., animals were disturbed by animal caretaker during the period between the training and test sessions) or by olfactory cues left by other mice and not well cleaned in between animals and/or sessions.

5. Very rarely, the mouse can jump out of the maze. To prevent these events, a transparent plastic cover can be placed above the maze.

\section{Acknowledgments}

We thank Delphine Gonzales, Nathalie Aubailly, and all the personnel of the Animal Facility of the NeuroCentre Magendie for mouse care as well as the personnel from the Parc de Recerca Biomèdica de Barcelona (PRBB). We would also like to thank the people who have performed experimental work using this task. This work was supported by INSERM (to G.M.), EU-FP7 (FP7PEOPLE-2013-IEF-623638 to A.B.-G.), French State/Agence Nationale de la Recherche (ANR-10IDEX-03-02 to A.B-G), and MINECO from AEI (RYC-2017-21776) to A.B-G.

\section{Competing interests}

The authors declare no competing interests.

\section{References}

1. Aloisi, E., Le Corf, K., Dupuis, J., Zhang, P., Ginger, M., Labrousse, V., Spatuzza, M., Georg Haberl, M., Costa, L., Shigemoto, R., Tappe-Theodor, A., Drago, F., Vincenzo Piazza, P., Mulle, C., Groc, L., Ciranna, L., Catania, M. V. and Frick, A. (2017). Altered surface mGluR5 dynamics provoke synaptic NMDAR dysfunction and cognitive defects in Fmr1 knockout mice. Nat Commun 8(1): 1103.

2. Aso, E., Palomer, E., Juves, S., Maldonado, R., Munoz, F. J. and Ferrer, I. (2012). $\underline{\text { CB1 agonist }}$ ACEA protects neurons and reduces the cognitive impairment of AbetaPP/PS1 mice. $J$ Alzheimers Dis 30(2): 439-459.

3. Bevins, R. A. and Besheer, J. (2006). Object recognition in rats and mice: a one-trial nonmatching-to-sample learning task to study 'recognition memory'. Nat Protoc 1(3): 1306-1311.

4. Broadbent, N. J., Gaskin, S., Squire, L. R. and Clark, R. E. (2010). Object recognition memory and the rodent hippocampus. Learn Mem 17(1): 5-11.

5. Busquets-Garcia, A., Gomis-Gonzalez, M., Guegan, T., Agustin-Pavon, C., Pastor, A., Mato, S., Perez-Samartin, A., Matute, C., de la Torre, R., Dierssen, M., Maldonado, R. and Ozaita, A. (2013). Targeting the endocannabinoid system in the treatment of fragile X syndrome. Nat Med 19(5): 603-607. 
6. Busquets-Garcia, A., Gomis-Gonzalez, M., Salgado-Mendialdua, V., Galera-Lopez, L., Puighermanal, E., Martin-Garcia, E., Maldonado, R. and Ozaita, A. (2018). Hippocampal protein kinase $C$ signaling mediates the short-term memory impairment induced by delta9tetrahydrocannabinol. Neuropsychopharmacology 43(5): 1021-1031.

7. Busquets-Garcia, A., Gomis-Gonzalez, M., Srivastava, R. K., Cutando, L., Ortega-Alvaro, A., Ruehle, S., Remmers, F., Bindila, L., Bellocchio, L., Marsicano, G., Lutz, B., Maldonado, R. and Ozaita, A. (2016). Peripheral and central CB1 cannabinoid receptors control stress-induced impairment of memory consolidation. Proc Natl Acad Sci U S A 113(35): 9904-9909.

8. Busquets-Garcia, A., Puighermanal, E., Pastor, A., de la Torre, R., Maldonado, R. and Ozaita, A. (2011). Differential role of anandamide and 2-arachidonoylglycerol in memory and anxietylike responses. Biol Psychiatry 70(5): 479-486.

9. Dere, E., Huston, J. P. and De Souza Silva, M. A. (2007). The pharmacology, neuroanatomy and neurogenetics of one-trial object recognition in rodents. Neurosci Biobehav Rev 31(5): 673704.

10. Ennaceur, A. (2010). One-trial object recognition in rats and mice: methodological and theoretical issues. Behav Brain Res 215(2): 244-254.

11. Ennaceur, A. and Delacour, J. (1988). A new one-trial test for neurobiological studies of memory in rats. 1: Behavioral data. Behav Brain Res 31(1): 47-59.

12. Hale, M. W., Hay-Schmidt, A., Mikkelsen, J. D., Poulsen, B., Shekhar, A. and Lowry, C. A. (2008). Exposure to an open-field arena increases c-Fos expression in a distributed anxiety-related system projecting to the basolateral amygdaloid complex. Neuroscience 155(3): 659-672.

13. Hebert-Chatelain, E., Desprez, T., Serrat, R., Bellocchio, L., Soria-Gomez, E., Busquets-Garcia, A., Pagano Zottola, A. C., Delamarre, A., Cannich, A., Vincent, P., Varilh, M., Robin, L. M., Terral, G., Garcia-Fernandez, M. D., Colavita, M., Mazier, W., Drago, F., Puente, N., Reguero, L., Elezgarai, I., Dupuy, J. W., Cota, D., Lopez-Rodriguez, M. L., Barreda-Gomez, G., Massa, F., Grandes, P., Benard, G. and Marsicano, G. (2016). A cannabinoid link between mitochondria and memory. Nature 539(7630): 555-559.

14. Oliveira da Cruz, J. F., Busquets-Garcia, A., Zhao, Z., Varilh, M., Lavanco, G., Bellocchio, L., Robin, L., Cannich, A., Julio-Kalajzić, F., Drago, F., Marsicano, G. and Soria-Gomez, E. (2019). Specific hippocampal interneurons shape consolidation of recognition memory. bioRxiv: 842021.

15. Mathis, A., Mamidanna, P., Cury, K. M., Abe, T., Murthy, V. N., Mathis, M. W. and Bethge, M. (2018). DeepLabCut: markerless pose estimation of user-defined body parts with deep learning. Nat Neurosci 21(9): 1281-1289.

16. Nath, V., Schilling, K. G., Parvathaneni, P., Hansen, C. B., Hainline, A. E., Huo, Y., Blaber, J. A., Lyu, I., Janve, V., Gao, Y., Stepniewska, I., Anderson, A. W. and Landman, B. A. (2019). Deep learning reveals untapped information for local white-matter fiber reconstruction in diffusionweighted MRI. Magn Reson Imaging 62: 220-227. 
17. Puighermanal, E., Busquets-Garcia, A., Gomis-Gonzalez, M., Marsicano, G., Maldonado, R. and Ozaita, A. (2013). Dissociation of the pharmacological effects of THC by mTOR blockade. Neuropsychopharmacology 38(7): 1334-1343.

18. Puighermanal, E., Marsicano, G., Busquets-Garcia, A., Lutz, B., Maldonado, R. and Ozaita, A. (2009). Cannabinoid modulation of hippocampal long-term memory is mediated by mTOR signaling. Nat Neurosci 12(9): 1152-1158.

19. Robin, L. M., Oliveira da Cruz, J. F., Langlais, V. C., Martin-Fernandez, M., Metna-Laurent, M., Busquets-Garcia, A., Bellocchio, L., Soria-Gomez, E., Papouin, T., Varilh, M., Sherwood, M. W., Belluomo, I., Balcells, G., Matias, I., Bosier, B., Drago, F., Van Eeckhaut, A., Smolders, I., Georges, F., Araque, A., Panatier, A., Oliet, S. H. R. and Marsicano, G. (2018). Astroglial CB1 receptors determine synaptic $D$-serine availability to enable recognition memory. Neuron 98(5): 935-944 e935.

20. Sik, A., van Nieuwehuyzen, P., Prickaerts, J. and Blokland, A. (2003). Performance of different mouse strains in an object recognition task. Behav Brain Res 147(1-2): 49-54.

21. Winters, B. D., Forwood, S. E., Cowell, R. A., Saksida, L. M. and Bussey, T. J. (2004). Double dissociation between the effects of peri-postrhinal cortex and hippocampal lesions on tests of object recognition and spatial memory: heterogeneity of function within the temporal lobe. $J$ Neurosci 24(26): 5901-5908.

22. Winters, B. D., Saksida, L. M. and Bussey, T. J. (2008). Object recognition memory: neurobiological mechanisms of encoding, consolidation and retrieval. Neurosci Biobehav Rev 32(5): 1055-1070. 